\title{
Treatment of Non-Alcoholic Fatty Liver Disease
}

\author{
Antonia Scherer ${ }^{a, b}$ Jean-François Dufour ${ }^{a, b}$ \\ ${ }^{a}$ Department of Clinical Research, Hepatology, and b University Clinic for Visceral Surgery and Medicine, Inselspital, \\ University of Bern, Bern, Switzerland
}

\section{Key Words}

Antioxidants · Drug treatments - Lifestyle changes .

Non-alcoholic steatohepatitis treatment · Transcription

factor agonists

\begin{abstract}
Non-alcoholic fatty liver disease (NAFLD) encompasses a spectrum of conditions from steatosis to cirrhosis and hepatocellular carcinoma. Steatosis is a benign reversible condition, which does not need treatment. Cirrhosis and hepatocellular carcinoma are the end stages of any chronic liver disease and do not have etiology-specific treatments. In this chapter, we will review treatment options for non-alcoholic steatohepatitis, which is the progressive form of NAFLD. Basically there are 2 strategies, the first of which is to address lifestyle and the second to use medication. The first approach is the most physiologic, the least expensive, but is also the most difficult to implement. The second approach, which should help patients who failed the first approach, is at the advanced clinical research stage.
\end{abstract}

\section{Lifestyle Changes}

Non-alcoholic steatohepatitis (NASH) occurs with such frequency because it is the consequence of our physiology being unable to adapt to our new living conditions, that is, the evolution selected for organisms able to store energy to survive famine. Nowadays, it is no longer a necessity for us to move to find food, as it would have been for our ancestors. We are constantly exposed to high energy and high caloric nutrients and overweight concerns a significant proportion of the population. Weight loss improves transaminases. In a prospective, longitudinal cohort study, a correlation between weight changes and modification of transaminases was found [1]. Patients who gained $>5 \%$ of body weight had increased transaminases, whereas those who lost $>5 \%$ of body weight saw an improvement in their transaminases. More than 5\% weight reduction was also associated with a decrease in serum triglycerides and fasting blood glucose, an increase in HDL cholesterol as well as decreased blood pressure. Harrison et al. [2] reported that losing $\geq 5 \%$ of body weight over 9 months was enough to improve insulin resistance as well

\section{KARGER}

E-Mail karger@karger.com www.karger.com/ddi
(C) 2016 S. Karger AG, Basel

0257-2753/16/0345-0027\$39.50/0
Prof. Jean-François Dufour

Department of Clinical Research

University Clinic for Visceral Surgery and Medicine

Inselspital, CH-3010 Bern (Switzerland)

E-Mail jean-francois.dufour@ikp.unibe.ch 
as steatosis and that a weight loss $\geq 9 \%$ was additionally associated with less hepatocyte ballooning, less inflammation and a decrease in non-alcoholic fatty liver disease (NAFLD) activity score. This was confirmed in a randomized controlled trial where there was a correlation between improvement of NAFLD activity score in the histology and the percentage of weight change [3]. People, who achieved a mean weight reduction of $8.7 \mathrm{~kg}$ due to lifestyle interventions during a 48-week period, could reduce their overall NASH activity score by $\geq 3$ points. Huang et al. [4] also showed an improvement in histology for NASH patients due to a 1-year dietary program.

Physical activity reduces steatosis in patients with NAFLD [5] even when it is not enough to change body weight [6] or to improve ALT [7], but according to St. George et al. [8], it does improve gamma GT significantly. Hallsworth et al. [9] also showed an improvement of steatosis in sedentary adults through resistance exercise, even at a level where it does not have an impact on body weight. In a study by $\mathrm{Oh}$ et al. [10], 169 obese men were enrolled in a 3-week weight reduction program. Those with physical activity of $>4$ h per week not only had an improvement of the hepatic steatosis, they also showed an improvement of hepatocellular apoptosis and hepatic stiffness.

With regards to diet, omega-3 polyunsaturated fatty acids have been suggested to improve steatosis and AST, but not ALT [11]. In a phase II randomized controlled trial though, no significant effects of ethyl-eicosapentaenoic acid on histologic features of NASH were reported [12]. Monounsaturated fatty acids have been reported in a controlled randomized study over 8 weeks to improve liver fat significantly, which was measured by proton nuclear magnetic resonance spectroscopy in patients with type 2 diabetes [13]. Regarding the benefit of a Mediterranean diet with a high consumption of monounsaturated fatty acids, Ryan et al. [14] performed a randomized crossover study, which also demonstrated a decrease in liver fat and an improvement in insulin sensitivity. Machado et al. [15] found out that trans- and saturated fatty acids correlate with oxidative stress markers such as 4-hydroxynonenal. In a rather provocative study, Kechagias et al. [16] placed 18 healthy individuals on a high caloric fast food-based diet for 4 weeks. They gained 6.4 $\mathrm{kg}$ and most of them had abnormal ALT at the end of the study. This clarifies the negative effects of excessive nutrition.

Where sodas are concerned, fructose reduces the satiety sensation; it is taken up into hepatocytes where it increases lipogenesis as well as the production of reactive oxygen species and reduces lipid oxidation [17]. Additionally, fructose has been shown to promote fibrosis as well as inflammation and hepatocyte ballooning in subjects older than 48 years [18].

There are more and more epidemiology studies showing the beneficial effects of coffee. In a large survey and multivariate analysis, low caffeine intake was found to be an independent predictor for NAFLD, after adjustment for race, gender and metabolic syndrome components [19]. Coffee consumption was lower in patients with more fibrosis, which allows the assumption of regular coffee having a protective role against NAFLD [20].

Provocatively, no alcohol is worse than a little bit of alcohol. In a study conducted by the NASH Clinical Research Network, it was discovered that NASH, fibrosis, ballooning, Mallory bodies and inflammation in histology occurred more frequently in non-drinkers than moderate drinkers [21].

Smoking has also been associated with NAFLD [22] and there are data showing that cannabis promotes liver fibrosis in patients with chronic hepatitis C [23]. Finally, sleep duration is also important. Less than $5 \mathrm{~h}$ of sleep elevates the risk of NAFLD in middle-aged people significantly and insufficient sleep has also been associated with an elevated risk for a fatty liver index $\geq 60$ [24].

Based on this evidence, it is clear that the first approach for patients with NASH is to review their habits and to discuss strategies with them that improve their lifestyles. Unfortunately, changing habits is difficult and even if it is the least expensive, the safest and the most effective treatment option for NASH, it is one of the least successful in practice. Recommendations to perform exercise, eat differently and lose weight often remain wishful thinking.

\section{Drug Treatments}

\section{Antioxidants}

In the past, antioxidative medication was discussed as a treatment of NASH. In randomized controlled trials, however, ursodeoxycholic acid was unconvincing as it was ineffective as monotherapy at the usual dose [25]. Thiazolidinediones, which function as PPAR $\gamma$ agonists, did show positive effects in patients with NASH in randomized controlled trials, but unfortunately, they result in significant weight gain [26].

The systematic review of the network meta-analysis comparing the effectiveness of pharmacologic interventions for NASH found that pentoxifylline, which is an inhibitor of TNF- $\alpha$, may have an anti-fibrotic effect [27]. A 
meta-analysis confirmed possible advantages of pentoxifylline in patients with NASH [28].

A randomized controlled trial performed with nondiabetic adults with NASH found that vitamin $\mathrm{E}$ for about 2 years led to a significant improvement in histologic lesions [29]. These results were duplicated in a pediatric trial [30], showing less hepatocellular ballooning in children who were treated with vitamin E. Singh et al. [27] also reported a positive effect on ballooning by vitamin $\mathrm{E}$. In a pooled analysis, it was suggested that vitamin E might also have a positive effect on histology in patients with diabetes and NASH (K. Kowdley et al. AASLD 2015, Abstract 107). However, they could neither confirm an association between vitamin $\mathrm{E}$ and changes in serum lipids nor an association with cardiac events. In other trials, however, vitamin $\mathrm{E}$ has been reported to be associated with cardiovascular events [31]. This is an issue in patients with NASH since they are already at risk of cardiovascular events. In addition, the extended follow-up of the SELECT trial demonstrated a significant association between vitamin $\mathrm{E}$ and an increased risk of developing prostate cancer among healthy men [32]. These aspects unfortunately decrease the attractiveness of an otherwise inexpensive, safe, well-tolerated and (probably) effective $\mathrm{NASH}$ treatment. It emphasizes the need for further trials on vitamin E, especially on its efficacy in diabetic patients as well as other possible treatments for NASH.

\section{Transcription Factor Agonists}

Obeticholic acid is a semi-synthetic bile acid analog and a ligand for the farnesoid X receptor (FXR) [33]. When bound to the FXR, it suppresses bile acid synthesis from cholesterol as well as hepatic lipid synthesis [34]. It increases peripheral clearance of VLDL and accelerates reverse cholesterol transport $[34,35]$. In a randomized controlled trial [36], obeticholic acid ( $25 \mathrm{mg}$ /day) was given to patients with NASH for 72 weeks. Eight centers in the US participated. The trial was halted when an interim analysis based on 140 of the 280 planned end of treatment biopsies found superiority of obeticholic acid compared with placebo for the primary outcome. The primary outcome was determined by a decrease in NAFLD activity score of 2 points without worsening of fibrosis. This was met by $45 \%$ of patients on obeticholic acid compared with $21 \%$ in the placebo group. Moreover, a greater number of patients assigned to obeticholic acid had an improvement in fibrosis, hepatocellular ballooning, steatosis and lobular inflammation. The mean change in NAFLD activity score was greater in patients treated with obeticholic acid. There was a significant reduction in the liver tests (such as ALAT, ASAT and $\gamma$-GT) except for alkaline phosphatase. Obeticholic acid treatment was also associated with weight loss. However, in patients with NASH, obeticholic acid led to higher cholesterol, higher LDL and decreased HDL cholesterol. Moreover, pruritus occurred in $23 \%$ of patients treated with obeticholic acid as compared with only $6 \%$ in the placebo group. Therefore, the long-term safety of obeticholic acid needs to be addressed for further clarification.

Elafibranor is a dual PPAR $\alpha$ and PPAR $\delta$ agonist. This drug has been tested in an international, phase II, randomized controlled trial [39]. It enrolled patients with a NAFLD activity score $>3$ from a large number of centers $(n=56)$ in Europe and the US. In an intention-to-treat analysis using the protocol definition of the primary end point, the study was negative. Using a new definition of the primary end point as a resolution of NASH without worsening of fibrosis, the result was statistically significant; $12 \%$ of patients in the placebo group achieved this new primary end point and $19 \%$ in the elafibranor group. The effect was stronger in patients with a NAFLD activity score $>4$ and in those with fibrosis. Importantly, this drug had beneficial effects on lipids with improvement of triglycerides, decrease in total cholesterol, decrease in non-HDL cholesterol and increase in HDL cholesterol. Furthermore, an improvement in insulin sensitivity was detected, suggesting that elafibranor may be safe in patients with NASH. Clearly, a larger study needs to duplicate these findings.

\section{Other Targets}

Other targets might be considered in the treatment of patients with NASH. Hepatocellular apoptosis is important for the progression of fibrosis. Anti-apoptotic drugs might prove useful in the treatment of NASH. Emricasan targets apoptosis through inhibition of caspases, and it is currently being evaluated in non-cirrhotic patients with NASH [37]. However, long-term safety of such suppression of apoptosis needs to be evaluated, as cancer is the second most frequent cause of death in NASH patients.

Patients with NASH are at risk of developing cirrhosis, which comes from deposition of fibrotic tissues in the liver. Therapeutic strategies aimed at preventing fibrosis might also work in patients with NASH. Simtuzumab is an antibody against lysine oxidants and its efficacy is currently being tested [37]. As an anti-fibrotic treatment, it may have a place in the future NASH treatment algorithm.

Some chemokine ligands and their receptors, such as CCR2 and CCR5, are upregulated during NASH, which results in liver inflammation, fibrosis and steatosis [38]. Cenicriviroc is a dual antagonist of CCR2 and CCR5, 
which is currently being tested [37]. By targeting chemokines, this approach may also have potential in the treatment of NASH.

\section{Conclusion}

What the field desperately needs is a positive definition of NASH. NASH implies that alcohol has been excluded as a cause of the disease and that one has a histology showing the typical lesions of the disease. It is crucial that we better understand the pathophysiologic mechanisms underlying this disease in order to have a positive non-invasive definition, which will allow the selection of more homogenous groups of treatments as well as better definitions of end points for clinical trials.

\section{Disclosure Statement}

Advisory committees: Abbvie, Bayer, BMS, Genfit, Gilead Science, Intercept, Merck, Novartis, Sillagen. Speaking and teaching: Abbvie, Bayer, Gilead Science. Unsrestricted research grant: Bayer.

\section{References}

1 Suzuki A, Lindor K, St Saver J, et al: Effect of changes on body weight and lifestyle in nonalcoholic fatty liver disease. J Hepatol 2005;43: 1060-1066.

2 Harrison SA, Fecht W, Brunt EM, et al: Orlistat for overweight subjects with nonalcoholic steatohepatitis: a randomized, prospective trial. Hepatology 2009;49:80-86.

3 Promrat K, Kleiner DE, Niemeier HM, et al: Randomized controlled trial testing the effects of weight loss on nonalcoholic steatohepatitis. Hepatology 2010;51:121129.

4 Huang MA, Greenson JK, Chao C, et al: One-year intense nutritional counseling results in histological improvement in patients with non-alcoholic steatohepatitis: a pilot study. Am J Gastroenterol 2005; 100:10721081.

5 Wong VW, Chan RS, Wong GL, et al: Community-based lifestyle modification programme for non-alcoholic fatty liver disease: a randomized controlled trial. J Hepatol 2013; 59:536-542.

6 Sullivan S, Kirk EP, Mittendorfer B, et al: Randomized trial of exercise effect on intrahepatic triglyceride content and lipid kinetics in nonalcoholic fatty liver disease. Hepatology 2012;55:1738-1745.

7 Keating SE, Hackett DA, George J, et al: Exercise and non-alcoholic fatty liver disease: a systematic review and meta-analysis. J Hepatol 2012;57:157-166.

8 St George A, Bauman A, Johnston A, et al: Independent effects of physical activity in patients with nonalcoholic fatty liver disease. Hepatology 2009;50:68-76.

9 Hallsworth K, Fattakhova G, Hollingsworth $\mathrm{KG}$, et al: Resistance exercise reduces liver fat and its mediators in non-alcoholic fatty liver disease independent of weight loss. Gut 2011; 60:1278-1283.

10 Oh S, Shida T, Yamagishi K, et al: Moderate to vigorous physical activity volume is an important factor for managing nonalcoholic fatty liver disease: a retrospective study. Hepatology 2015;61:1205-1215.
11 Parker HM, Johnson NA, Burdon CA, et al: Omega-3 supplementation and non-alcoholic fatty liver disease: a systematic review and meta-analysis. J Hepatol 2012;56:944-951.

12 Sanyal AJ, Abdelmalek MF, Suzuki A, et al: No significant effects of ethyl-eicosapentanoic acid on histologic features of nonalcoholic steatohepatitis in a phase 2 trial. Gastroenterology 2014;147:377-384.

13 Bozzetto L, Prinster A, Annuzzi G, et al: Liver fat is reduced by an isoenergetic MUFA diet in a controlled randomized study in type 2 diabetic patients. Diabetes Care 2012;35: 1429-1435.

14 Ryan MC, Itsiopoulos C, Thodis T, et al: The mediterranean diet improves hepatic steatosis and insulin sensitivity in individuals with non-alcoholic fatty liver disease. J Hepatol 2013;59:138-143.

15 Machado MV, Ravasco P, Jesus L, et al: Blood oxidative stress markers in non-alcoholic steatohepatitis and how it correlates with diet. Scand J Gastroenterol 2008;43:95-102.

16 Kechagias S, Ernersson A, Dahlqvist O, et al: Fast-food-based hyper-alimentation can induce rapid and profound elevation of serum alanine aminotransferase in healthy subjects. Gut 2008;57:649-654.

17 Basaranoglu M, Basaranoglu G, Sabuncu T, et al: Fructose as a key player in the development of fatty liver disease. World J Gastroenterol 2013;19:1166-1172.

18 Abdelmalek MF, Suzuki A, Guy C, et al: Increased fructose consumption is associated with fibrosis severity in patients with nonalcoholic fatty liver disease. Hepatology 2010; 51:1961-1971.

19 Birerdinc A, Stepanova M, Pawloski L, et al: Caffeine is protective in patients with nonalcoholic fatty liver disease. Aliment Pharmacol Ther 2012;35:76-82.

20 Anty R, Marjoux S, Iannelli A, et al: Regular coffee but not espresso drinking is protective against fibrosis in a cohort mainly composed of morbidly obese European women with NAFLD undergoing bariatric surgery. J Hepatol 2012;57:1090-1096.
21 Dunn W, Sanyal AJ, Brunt EM, et al: Modest alcohol consumption is associated with decreased prevalence of steatohepatitis in patients with non-alcoholic fatty liver disease (NAFLD). J Hepatol 2012;57:384-391.

22 Liu Y, Dai M, Bi Y, et al: Active smoking, passive smoking, and risk of nonalcoholic fatty liver disease (NAFLD): a population-based study in china. J Epidemiol 2013;23:115121.

23 Hézode C, Roudot-Thoraval F, Nguyen S, et al: Daily cannabis smoking as a risk factor for progression of fibrosis in chronic hepatitis $\mathrm{C}$. Hepatology 2005;42:63-71.

24 Kim CW, Yun KE, Jung HS, et al: Sleep duration and quality in relation to non-alcoholic fatty liver disease in middle-aged workers and their spouses. J Hepatol 2013;59:351-357.

25 Haedrich M, Dufour JF: UDCA for NASH: end of the story? J Hepatol 2011;54:856-858.

26 Mahady SE, Webster AC, Walker S, et al: The role of thiazolidinediones in non-alcoholic steatohepatitis - a systematic review and meta analysis. J Hepatol 2011;55:1383-1390.

27 Singh S, Khera R, Allen AM, et al: Comparative effectiveness of pharmacological interventions for nonalcoholic steatohepatitis: a systematic review and network meta-analysis. Hepatology 2015;62:1417-1432.

$28 \mathrm{Du}$ J, Ma YY, Yu CH, et al: Effects of pentoxifylline on nonalcoholic fatty liver disease: a meta-analysis. World J Gastroenterol 2014; 20:569-577.

29 Hoofnagle JH, Van Natta ML, Kleiner DE, et al; Non-Alcoholic Steatohepatitis Clinical Research Network (NASH CRN): Vitamin E and changes in serum alanine aminotransferase levels in patients with non-alcoholic steatohepatitis. Aliment Pharmacol Ther 2013;38: 134-143.

30 Lavine JE, Schwimmer JB, Van Natta ML, et al; Nonalcoholic Steatohepatitis Clinical Research Network: Effect of vitamin E or metformin for treatment of nonalcoholic fatty liver disease in children and adolescents: the TONIC randomized controlled trial. JAMA 2011;305:1659-1668. 
31 Lonn E, Bosch J, Yusuf S, et al; HOPE and HOPE-TOO Trial Investigators: Effects of long-term vitamin $\mathrm{E}$ supplementation on cardiovascular events and cancer. JAMA 2005; 293:1338-1347.

32 Klein EA, Thompson IM Jr, Tangen CM, et al: Vitamin $\mathrm{E}$ and the risk of prostate cancer: the selenium and vitamin $\mathrm{E}$ cancer prevention trial (SELECT). JAMA 2011;306:1549-1556.

33 Pellicciari R, Fiorucci S, Camaioni E, et al: 6alpha-ethyl-chenodeoxycholic acid (6-ECDCA), a potent and selective FXR agonist endowed with anticholestatic activity. J Med Chem 2002; 45:3569-3572.
34 Modica S, Gadaleta RM, Moschetta A: Deciphering the nuclear bile acid receptor FXR paradigm. Nucl Recept Signal 2010; 8:e005.

35 Lambert G, Amar MJ, Guo G, et al: The farnesoid X-receptor is an essential regulator of cholesterol homeostasis. J Biol Chem 2003; 278:2563-2570.

36 Neuschwander-Tetri BA, Loomba R, Sanyal AJ, et al; NASH Clinical Research Network: Farnesoid X nuclear receptor ligand obeticholic acid for non-cirrhotic, non-alcoholic steatohepatitis (FLINT): a multicentre, randomised, placebo-controlled trial. Lancet 2015;385:956-965.

37 Musso G, Cassader M, Gambino R: Non-alcoholic steatohepatitis: emerging molecular targets and therapeutic strategies. Nat Rev Drug Discov 2016;15:249-274.
38 Marra F, Tacke F: Roles for chemokines in liver disease. Gastroenterology 2014;147: 577-594.e1.

39 Ratziu V, Harrison SA, Francque S, Bedossa P, Lehert P, Serfaty L, Romero-Gomez M, Boursier J, Abdelmalek M, Caldwell S, Drenth J, Anstee QM, Hum D, Hanf R, Roudot A, Megnien S, Staels B, Sanyal A; GOLDEN-505 Investigator Study Group: Elafibranor, an agonist of the peroxisome proliferator-activated receptor- $\alpha$ and $-\delta$, induces resolution of nonalcoholic steatohepatitis without fibrosis worsening. Gastroenterology 2016;150:11471159. 